\title{
Defining and Analysing Resource Assignments in Business Processes with RAL ${ }^{\star}$
}

\author{
Cristina Cabanillas, Manuel Resinas, and Antonio Ruiz-Cortés \\ Universidad de Sevilla, Spain \\ \{cristinacabanillas, resinas, aruiz\}@us.es
}

\section{Summary of the Contribution}

Business processes (BPs) are often analysed in terms of control flow, temporal constraints, data and resources. From all of these aspects, resources have received much less attention than other aspects, specially control flow. Even the standard $\mathrm{BP}$ modelling notation (BPMN) does not provide concrete definitions for the resource-related concepts [1]. However, the participation of people in BPs is of utmost importance, both to supervise the execution of automatic activities and to carry out software-aided and/or manual tasks. Therefore, they should be considered when designing and modelling the BPs used in an organization.

In this paper we face human-resource management (resource management for short) in BP models. Firstly, we deal with the assignment of resources to the activities of a BP model, aiming at easing and improving the way resources can be associated with BP activities. Some approaches addressing a similar purpose have been introduced in the last years [2-4], but they are in general either too complex to be used by technically unskilled people, or not expressive enough to provide powerful resource management in workflows (WFs) and BPs.

Another substantial shortage in many existing approaches related to resource management in WFs and BPs (e.g. in the default resource management mechanism provided by BPMN 2.0), is that they do not bridge the gap between organizational models and BP models, which means they do not actually relate both elements, thus treating them separately. Our second goal in this work is to come up with a solution that narrows this gap, at the same time as it allows us to analyse the resource assignments associated to the BP activities. With such a solution, organizations can benefit from the automation of work in different directions, to be named:

- The inference of interesting information, such as: (i) the potential performers of each BP activity, i.e., the set of people that meet the resource-related constraints imposed in the model (by means of assignment expressions associated to the BP activities); or (ii) the potential set of activities each person

\footnotetext{
* This work has been published in the 9th International Conference on Service Oriented Computing (ICSOC 2011). It has been partially supported by the European Commission (FEDER), Spanish Government under project SETI (TIN2009-07366); and projects THEOS (TIC-5906) and ISABEL (P07-TIC-2533) funded by the Andalusian Local Government.
} 
of an organization can be allocated at run time. This kind of information may be beneficial for an organization in several ways. For instance, in the previous case: the former benefits the person in charge of resource allocation, since it increases the information available to allocate tasks to resources when the BP is executed; and the latter provides an employee-oriented vision, informing about the possible workload of each employee and, hence, allowing reacting in time to avoid having people overburderned with work.

- The detection of inconsistencies between the resource assignments associated to activities of a BP model and the structure of the organization where it is used, e.g. non-existent roles or persons.

The contribution of this paper is, hence, twofold:

1. On the one hand, we have developed RAL (Resource Assignment Language), a DSL (Domain Specific Language) to express resource assignments in BP activities. It is based on an organizational metamodel proposed by Russell et al. [5], which considers the company structured according to persons, organizational units, roles and a hierarchy of positions. RAL was previously introduced in [6]. Its expressiveness has been tested using the group of creation patterns of the well-known Workflow Resource Patterns [5].

2. On the other hand, we provide a semantic mapping of RAL expressions into description logics (DLs). This allows us to use DL reasoners to automatically infer information about resource management from RAL assignments, and detect assignment inconsistencies, as explained above.

RAL Solver has been developed as a plugin for Oryx [7], to prove the use of RAL expressions in BP models and the benefits of its DL-based semantics. More information about the language and its analysis features can be found at http://www.isa.us.es/cristal.

\section{References}

1. "BPMN 2.0," recommendation, OMG, 2011.

2. A. Awad, A. Grosskopf, A. Meyer, and M. Weske, "Enabling Resource Assignment Constraints in BPMN," tech. rep., BPT, 2009.

3. C. Wolter, P. Miseldine, and C. Meinel, "Verification of Business Process Entailment Constraints Using SPIN," in Engineering Secure Software and Systems, vol. 5429 of Lecture Notes in Computer Science, pp. 1-15, Springer Berlin / Heidelberg, 2009.

4. M. Strembeck and J. Mendling, "Modeling process-related RBAC models with extended UML activity models," Inf. Softw. Technol., vol. 53, pp. 456-483, 2011.

5. N. Russell, W. M. P. van der Aalst, A. H. M. ter Hofstede, and D. Edmond, "Workflow resource patterns: Identification, representation and tool support," in CAiSE, pp. 216-232, 2005.

6. C. Cabanillas, M. Resinas, and A. Ruiz-Cortés, "RAL: A High-Level User-Oriented Resource Assignment Language for Business Processes," in Business Process Management Workshops (BPD'11), pp. 50-61, 2012.

7. G. Decker, H. Overdick, and M. Weske, "Oryx - an open modeling platform for the BPM community," in Business Process Management (BPM), pp. 382-385, SpringerVerlag, 2008. 